Discrete Comput Geom 35:493-512 (2006)

DOI: $10.1007 / \mathrm{s} 00454-005-1225-8$

\title{
Globally Linked Pairs of Vertices in Equivalent Realizations of Graphs*
}

\author{
Bill Jackson, ${ }^{1}$ Tibor Jordán, ${ }^{2}$ and Zoltán Szabadka ${ }^{2}$ \\ ${ }^{1}$ School of Mathematical Sciences, Queen Mary, University of London, \\ Mile End Road, London E1 4NS, England \\ B.Jackson@qmul.ac.uk \\ ${ }^{2}$ Department of Operations Research, Eötvös University, \\ Pázmány Péter sétány 1/C, 1117 Budapest, Hungary \\ \{jordan,sinus\}@cs.elte.hu.edu
}

\begin{abstract}
A two-dimensional framework $(G, p)$ is a graph $G=(V, E)$ together with a map $p: V \rightarrow \mathbb{R}^{2}$. We view $(G, p)$ as a straight line realization of $G$ in $\mathbb{R}^{2}$. Two realizations of $G$ are equivalent if the corresponding edges in the two frameworks have the same length. A pair of vertices $\{u, v\}$ is globally linked in $G$ if the distance between the points corresponding to $u$ and $v$ is the same in all pairs of equivalent generic realizations of $G$. The graph $G$ is globally rigid if all of its pairs of vertices are globally linked. We extend the characterization of globally rigid graphs given by the first two authors [13] by characterizing globally linked pairs in $M$-connected graphs, an important family of rigid graphs. As a byproduct we simplify the proof of a result of Connelly [6] which is a key step in the characterization of globally rigid graphs. We also determine the number of distinct realizations of an $M$ connected graph, each of which is equivalent to a given generic realization. Bounds on this number for minimally rigid graphs were obtained by Borcea and Streinu in [3].
\end{abstract}

\section{Introduction}

We consider finite graphs without loops, multiple edges or isolated vertices. A $d$ dimensional framework is a pair $(G, p)$, where $G=(V, E)$ is a graph and $p$ is a map from $V$ to $\mathbb{R}^{d}$. We consider the framework to be a straight line realization of $G$ in $\mathbb{R}^{d}$.

* Bill Jackson was supported by the Finite Structures Project of the Rényi Institute of Mathematics of the Hungarian Academy of Sciences, within the 6th Framework Programme of the EU. Tibor Jordán was supported by the MTA-ELTE Egerváry Research Group on Combinatorial Optimization, and the Hungarian Scientific Research Fund Grant No. T049671 and T037547. 
Two frameworks $(G, p)$ and $(G, q)$ are equivalent if $\|p(u)-p(v)\|=\|q(u)-q(v)\|$ holds for all pairs $u, v$ with $u v \in E$, where $\|\cdot\|$ denotes the Euclidean norm in $\mathbb{R}^{d}$. Frameworks $(G, p)$ and $(G, q)$ are congruent if $\|p(u)-p(v)\|=\|q(u)-q(v)\|$ holds for all pairs $u, v$ with $u, v \in V$. This is the same as saying that $(G, q)$ can be obtained from $(G, p)$ by an isometry of $\mathbb{R}^{d}$.

We say that $(G, p)$ is globally rigid if every framework which is equivalent to $(G, p)$ is congruent to $(G, p)$. The framework $(G, p)$ is rigid if there exists an $\varepsilon>0$ such that if $(G, q)$ is equivalent to $(G, p)$ and $\|p(u)-q(u)\|<\varepsilon$ for all $v \in V$ then $(G, q)$ is congruent to $(G, p)$. Intuitively, this means that if we think of a $d$-dimensional framework $(G, p)$ as a collection of bars and joints where points correspond to joints and each edge to a rigid bar joining its endpoints, then the framework is rigid if it has no non-trivial continuous deformations (see [8] and also Section 3.2 of [20]). It seems to be a hard problem to decide if a given framework is rigid or globally rigid. Indeed, Saxe [18] has shown that it is NP-hard to decide if even a one-dimensional framework is globally rigid. These problems become more tractable, however, if we assume that there are no algebraic dependencies between the coordinates of the points of the framework.

A framework $(G, p)$ is said to be generic if the set containing the coordinates of all its points is algebraically independent over the rationals. It is known [20] that rigidity of frameworks in $\mathbb{R}^{d}$ is a generic property, that is, the rigidity of $(G, p)$ depends only on the graph $G$ and not on the particular realization $p$, if $(G, p)$ is generic. We say that the graph $G$ is rigid in $\mathbb{R}^{d}$ if every (or, equivalently, if some) generic realization of $G$ in $\mathbb{R}^{d}$ is rigid.

The problem of characterizing when a graph is rigid in $\mathbb{R}^{d}$ has been solved for $d=1,2$. A graph is rigid in $\mathbb{R}$ if and only if it is connected. The characterization of rigid graphs in $\mathbb{R}^{2}$ is a result of Lovász and Yemini [15].

A similar situation holds for global rigidity: the problem of characterizing when a generic framework is globally rigid in $\mathbb{R}^{d}$ has also been solved for $d=1,2$. A onedimensional generic framework $(G, p)$ is globally rigid if and only if either $G$ is the complete graph on two vertices or $G$ is 2-connected. The characterization for $d=2$ follows from the following results. We say that $G$ is redundantly rigid in $\mathbb{R}^{d}$ if $G-e$ is rigid in $\mathbb{R}^{d}$ for all edges $e$ of $G$.

Theorem 1.1 [11]. Let $(G, p)$ be a generic framework in $\mathbb{R}^{d}$. If $(G, p)$ is globally rigid then either $G$ is a complete graph with at most $d+1$ vertices, or $G$ is $(d+1)$-connected and redundantly rigid in $\mathbb{R}^{d}$.

The Henneberg 1-extension operation [12] (on edge $x y$ and vertex $w$ ) deletes an edge $x y$ from a graph $G$ and adds a new vertex $z$ and new edges $z x, z y, z w$ for some vertex $w \in V(G)-\{x, y\}$. A key step in proving that the necessary conditions for global rigidity in Theorem 1.1 are also sufficient when $d=2$, is the following result of Connelly, see the Proof of Corollary 1.7 of [6].

Theorem 1.2 [6]. Suppose that $G$ can be obtained from $K_{4}$ by a sequence of 1 extensions and edge additions. Then every generic realization of $G$ in $\mathbb{R}^{2}$ is globally rigid. 


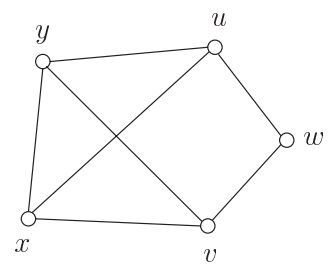

Fig. 1. A realization $(G, p)$ of a rigid graph $G$. The pair $\{u, v\}$ is globally linked in $(G, p)$.

The following recent result gives an inductive construction for graphs which are 3 -connected and redundantly rigid in $\mathbb{R}^{2}$.

Theorem 1.3 [13, Theorem 6.15]. Let $G$ be a 3-connected graph which is redundantly rigid in $\mathbb{R}^{2}$. Then $G$ can be obtained from $K_{4}$ by a sequence of 1-extensions and edge additions.

By observing that complete graphs are globally rigid, we obtain a complete characterization for globally rigid generic frameworks in $\mathbb{R}^{2}$.

Theorem 1.4 [6], [13]. Let $(G, p)$ be a two-dimensional generic framework. Then $(G, p)$ is globally rigid if and only if either $G$ is a complete graph on two or three vertices, or $G$ is 3-connected and redundantly rigid in $\mathbb{R}^{2}$.

It follows that global rigidity of frameworks in $\mathbb{R}^{d}$ is a generic property when $d=1,2$. It is not known whether this remains true for any $d \geq 3$. Following Connelly [5], we say that a graph $G$ is globally rigid in $\mathbb{R}^{d}$ if every (or equivalently when $1 \leq d \leq 2$, if some) generic realization of $G$ in $\mathbb{R}^{d}$ is globally rigid. We refer the reader to [10] and [20] for a detailed survey of the rigidity of $d$-dimensional frameworks.

In this paper we consider properties of two-dimensional generic frameworks which are weaker than global rigidity. We assume henceforth that $d=2$, unless specified otherwise. A pair of vertices $\{u, v\}$ in a framework $(G, p)$ is globally linked in $(G, p)$ if, in all equivalent frameworks $(G, q)$, we have $\|p(u)-p(v)\|=\|q(u)-q(v)\|$. The pair $\{u, v\}$ is globally linked in $G$ if it is globally linked in all generic frameworks $(G, p)$. Thus $G$ is globally rigid if and only if all pairs of vertices of $G$ are globally linked. Unlike global rigidity, however, "global linkedness" is not a generic property in $\mathbb{R}^{2}$. Figures 1 and 2 give an example of a pair of vertices in a rigid graph $G$ which is globally linked in one generic realization, but not in another. ${ }^{1}$

We first show that global linkedness is preserved by the 1-extension operation. More precisely we show that if $\{u, v\}$ is globally linked in $G=(V, E), w, x, y \in V, x y \in E$, and $G-x y$ is rigid, then $\{u, v\}$ is globally linked in the graph obtained from $G$ by a 1 -extension on edge $x y$ and vertex $w$. By using Theorem 1.1, we deduce that global rigidity is preserved by the 1-extension operation. This immediately gives Theorem 1.2

\footnotetext{
${ }^{1}$ Note that if $d=1$ then global linkedness is a generic property: $\{u, v\}$ is globally linked in $G$ if and only if $G$ has two openly disjoint $u v$-paths.
} 

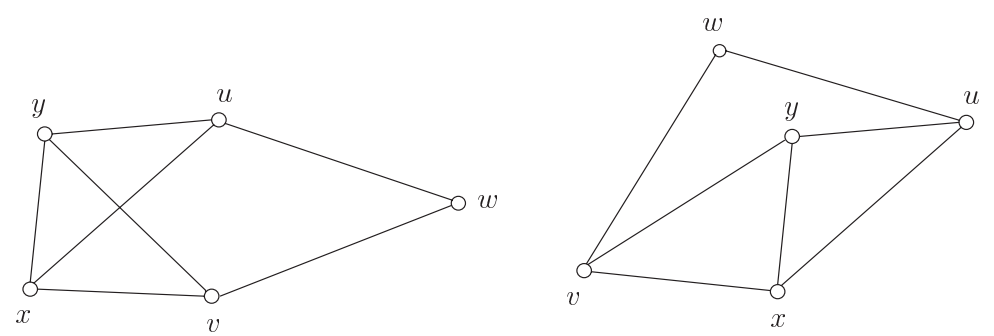

Fig. 2. Two equivalent realizations of the rigid graph $G$ of Fig. 1, which show that the pair $\{u, v\}$ is not globally linked in $G$.

and hence simplifies the proof of Theorem 1.4. (Connelly deduces Theorem 1.2 from a sufficient condition for the global rigidity of a $d$-dimensional framework in terms of the rank of its "stress matrix" [6, Theorem 1.5]. His proof of this sufficient condition uses some previous results from [4] together with other results from differential topology and the elimination theory of semi-algebraic sets.)

In the remainder of the paper we consider the following problems for a generic realization $(G, p)$ of a graph $G=(V, E)$ :

(a) Given $\{u, v\} \subset V$, when is $\{u, v\}$ globally linked in $(G, p)$ ?

(b) Given $v \in V$ and $U \subset V$, when is $v$ uniquely localizable with respect to $U$, that is to say, when is it true that every realization $(G, q)$ which is equivalent to $(G, p)$ and satisfies $p(u)=q(u)$ for all $u \in U$, must also satisfy $p(v)=q(v)$ ?

(c) Given $\{u, v\} \subset V$, when is $\{u, v\}$ globally loose in $G$, that is to say, when is it true that for all generic realizations $(G, p)$, there exists an equivalent realization $(G, q)$ which satisfies $\|p(u)-p(v)\| \neq\|q(u)-q(v)\|$ ?

(d) How many different realizations of $G$ are there which are each equivalent to $(G, p)$ ?

We use our result on 1-extensions to solve each of these problems for $M$-connected graphs, an important family of rigid graphs. Our results imply that the answer to each of the problems described in (a), (b) and (d) is generic when $G$ is $M$-connected, in the sense that the answer is the same for all generic realizations of $G$.

\section{The Rigidity Matroid}

The rigidity matroid of a graph $G$ is a matroid defined on the set of edges of $G$ which reflects the rigidity properties of all generic realizations of $G$. We need basic definitions and results on this matroid to define $M$-connected graphs and characterize global linkedness in these graphs.

Let $(G, p)$ be a realization of a graph $G=(V, E)$. The rigidity matrix of the framework $(G, p)$ is the matrix $R(G, p)$ of size $|E| \times 2|V|$, where, for each edge $v_{i} v_{j} \in E$, in the row corresponding to $v_{i} v_{j}$, the entries in the two columns corresponding to vertices $i$ and $j$ contain the two coordinates of $\left(p\left(v_{i}\right)-p\left(v_{j}\right)\right)$ and $\left(p\left(v_{j}\right)-p\left(v_{i}\right)\right)$, respectively, and the remaining entries are zeros. See [20] for more details. The rigidity matrix of 
$(G, p)$ defines the rigidity matroid of $(G, p)$ on the ground set $E$ by linear independence of rows of the rigidity matrix. Any two generic frameworks $(G, p)$ and $(G, q)$ have the same rigidity matroid. We call this the rigidity matroid $\mathcal{R}(G)=(E, r)$ of the graph $G$. We denote the rank of $\mathcal{R}(G)$ by $r(G)$. Gluck characterized rigid graphs in terms of their rank.

Theorem 2.1 [8]. Let $G=(V, E)$ be a graph. Then $G$ is rigid if and only if $r(G)=$ $2|V|-3$.

We say that a graph $G=(V, E)$ is $M$-independent if $E$ is independent in $\mathcal{R}(G)$. Knowing when subgraphs of $G$ are $M$-independent allows us to determine the rank of $G$. This can be accomplished using the following characterization of $M$-independent graphs due to Laman. For $X \subseteq V$, let $E_{G}(X)$ denote the set, and $i_{G}(X)$ the number, of edges in $G[X]$, that is, in the subgraph induced by $X$ in $G$.

Theorem 2.2 [14]. A graph $G=(V, E)$ is $M$-independent if and only if $i_{G}(X) \leq$ $2|X|-3$ for all $X \subseteq V$ with $|X| \geq 2$.

A graph $G=(V, E)$ is minimally rigid if $G$ is rigid, but $G-e$ is not rigid for all $e \in E$. Theorems 2.1 and 2.2 imply that $G$ is minimally rigid if and only if $G$ is $M$-independent and $|E|=2|V|-3$. Note that, if $G$ is rigid, then the edge sets of the minimally rigid spanning subgraphs of $G$ form the bases in the rigidity matroid of $G$.

A pair of vertices $\{u, v\}$ in a framework $(G, p)$ is linked in $(G, p)$ if there exists an $\varepsilon>0$ such that, if $(G, q)$ is equivalent to $(G, p)$ and $\|p(w)-q(w)\|<\varepsilon$ for all $w \in V$, then we have $\|p(u)-p(v)\|=\|q(u)-q(v)\|$. Using Theorems 2.1 and 2.2, it can be seen that this is a generic property and that $\{u, v\}$ is linked in a generic framework $(G, p)$ if and only if $G$ has a rigid subgraph $H$ with $\{u, v\} \subseteq V(H)$.

A compact characterization of all linked pairs can be deduced as follows. We define a rigid component of $G$ to be a maximal rigid subgraph of $G$. It is well known (see, e.g., Corollary 2.14 of [13]) that any two rigid components of $G$ intersect in at most one vertex and hence that the edge sets of the rigid components of $G$ partition the edges of $G$. Thus $\{u, v\}$ is linked in a generic framework $(G, p)$ if and only if $\{u, v\} \subseteq V(H)$ for some rigid component $H$ of $G$. Note that the rigid components of a graph can be determined in polynomial time, see for example [2].

\section{Generic Points and Quasi-Generic Frameworks}

In this section we prove some preliminary results on generic frameworks which we use in our proof that 1-extensions preserve global linkedness. A point $\mathbf{x} \in \mathbb{R}^{n}$ is generic if its components form an algebraically independent set over $\mathbb{Q}$.

Lemma 3.1. Let $f: \mathbb{R}^{n} \rightarrow \mathbb{R}^{m}$ by $f(\mathbf{x})=\left(f_{1}(\mathbf{x}), f_{2}(\mathbf{x}), \ldots, f_{m}(\mathbf{x})\right)$, where $f_{i}(\mathbf{x})$ is a polynomial with integer coefficients for all $1 \leq i \leq m$. Suppose that $\max _{\mathbf{x} \in \mathbb{R}^{n}}\left\{\right.$ rank $\left.\left.d f\right|_{\mathbf{x}}\right\}=$ $m$. If $\mathbf{p}$ is a generic point in $\mathbb{R}^{n}$, then $f(\mathbf{p})$ is a generic point in $\mathbb{R}^{m}$. 
Proof. Since $\mathbf{p}$ is generic, we have rank $\left.d f\right|_{\mathbf{p}}=m$. Relabelling if necessary, we may suppose that the first $m$ columns of $\left.d f\right|_{\mathbf{p}}$ are linearly independent. Let $\mathbf{p}=$ $\left(p_{1}, p_{2}, \ldots, p_{n}\right)$. Define $f^{\prime}: \mathbb{R}^{m} \rightarrow \mathbb{R}^{m}$ by $f^{\prime}\left(x_{1}, x_{2}, \ldots, x_{m}\right)=f\left(x_{1}, x_{2}, \ldots, x_{m}\right.$, $\left.p_{m+1}, \ldots, p_{n}\right)$. Let $\mathbf{p}^{\prime}=\left(p_{1}, p_{2}, \ldots, p_{m}\right)$. Then $f^{\prime}\left(\mathbf{p}^{\prime}\right)=f(\mathbf{p})$ and rank $\left.d f^{\prime}\right|_{\mathbf{p}^{\prime}}=m$.

Let $f^{\prime}\left(\mathbf{p}^{\prime}\right)=\left(\beta_{1}, \beta_{2}, \ldots, \beta_{m}\right)$. Suppose that $g\left(\beta_{1}, \beta_{2}, \ldots, \beta_{m}\right)=0$ for some polynomial $g$ with integer coefficients. Then $g\left(f_{1}(\mathbf{p}), f_{2}(\mathbf{p}), \ldots, f_{m}(\mathbf{p})\right)=0$. Since $\mathbf{p}$ is generic, we must have $g\left(f^{\prime}(\mathbf{x})\right)=0$ for all $\mathbf{x} \in \mathbb{R}^{m}$. By the inverse function theorem $f^{\prime}$ maps a sufficiently small open neighbourhood $U$ of $\mathbf{p}^{\prime}$ diffeomorphically onto $f^{\prime}(U)$. Thus $g(\mathbf{y})=g\left(f^{\prime}(\mathbf{x})\right)=0$ for all $\mathbf{y} \in f^{\prime}(U)$. Since $g$ is a polynomial map and $f^{\prime}(U)$ is an open subset of $\mathbb{R}^{m}$, we have $g \equiv 0$. Hence $f^{\prime}\left(\mathbf{p}^{\prime}\right)=f(\mathbf{p})$ is generic.

Given a point $\mathbf{p} \in \mathbb{R}^{n}$ we use $\mathbb{Q}(\mathbf{p})$ to denote the field extension of $\mathbb{Q}$ by the coordinates of $\mathbf{p}$. Given fields $K \subseteq L$ with $L$ a finitely generated field extension of $K$, the transcendence degree of $L$ over $K, t d[L: K]$, is the size of a largest subset of $L$ which is algebraically independent over $K$, see Section 18.1 of [19]. (It follows from the Steinitz exchange axiom, see Lemma 18.4 of [19] and Section 6.7 of [17], that this definition gives rise to a matroid on $L$, where the rank of a subset $S$ of $L$ is $t d[K(S): K]$.) We use $\tilde{K}$ to denote the algebraic closure of $K$. Note that each element of $\tilde{K}$ is a loop in the above mentioned matroid and hence $t d[\tilde{K}: K]=0$.

Lemma 3.2. Let $f: \mathbb{R}^{n} \rightarrow \mathbb{R}^{n}$ by $f(\mathbf{x})=\left(f_{1}(\mathbf{x}), f_{2}(\mathbf{x}), \ldots, f_{n}(\mathbf{x})\right)$, where $f_{i}(\mathbf{x})$ is a polynomial with integer coefficients for all $1 \leq i \leq n$. Suppose that $f(\mathbf{p})$ is a generic point in $\mathbb{R}^{n}$. Let $L=\mathbb{Q}(\mathbf{p})$ and $K=\mathbb{Q}(f(\mathbf{p}))$. Then $\tilde{K}=\tilde{L}$.

Proof. Since $f_{i}(\mathbf{x})$ is a polynomial with integer coefficients, we have $f_{i}(\mathbf{p}) \in L$ for all $1 \leq i \leq n$. Thus $K \subseteq L$. Since $f(\mathbf{p})$ is generic we have $t d[K: \mathbb{Q}]=n$. Since $K \subseteq L$ and $L=\mathbb{Q}(\mathbf{p})$ we have $t d[L: \mathbb{Q}]=n$. Thus $\tilde{K} \subseteq \tilde{L}$ and $t d[\tilde{K}: \mathbb{Q}]=n=$ $t d[\tilde{\tilde{L}}: \mathbb{Q}]$. Suppose $\tilde{K} \neq \tilde{L}$, and choose $\gamma \in \tilde{L}-\tilde{K}$. Then $\gamma$ is not algebraic over $K$ so $S=\left\{\gamma, f_{1}(\mathbf{p}), f_{2}(\mathbf{p}), \ldots, f_{n}(\mathbf{p})\right\}$ is algebraically independent over $\mathbb{Q}$. This contradicts the facts that $S \subseteq \tilde{L}$ and $t d[\tilde{L}: \mathbb{Q}]=n$.

A configuration $C$ is a set $\left\{p_{1}, p_{2}, \ldots, p_{n}\right\}$ of points in $\mathbb{R}^{2}$. We say that $C$ is generic if the point $\mathbf{p}=\left(p_{1}, p_{2}, \ldots, p_{n}\right) \in \mathbb{R}^{2 n}$ is generic. Two configurations $C$ and $C^{\prime}$ are congruent if there exists an isometry $T$ of $\mathbb{R}^{2}$ such that $T(C)=C^{\prime}$. We say that $C$ is quasi-generic if $C$ is congruent to a generic configuration, and that $C$ is in standard position if $p_{1}=(0,0)$ and $p_{2}=\left(0, y_{2}\right)$ for some $y_{2} \in \mathbb{R}$.

Let $G=(V, E)$ be a graph and let $(G, p)$ be a realization of $G$. Let $V=\left\{v_{1}, v_{2}, \ldots, v_{n}\right\}$ and $E=\left\{e_{1}, e_{2}, \ldots, e_{m}\right\}$. We can view $p$ as a point $\mathbf{p}=\left(p\left(v_{1}\right), p\left(v_{2}\right), \ldots, p\left(v_{n}\right)\right)$ in $\mathbb{R}^{2 n}$. We say that $(G, p)$ is quasi-generic or in standard position if $p(V)$ is, respectively, quasi-generic or in standard position. The rigidity map $f_{G}: \mathbb{R}^{2 n} \rightarrow \mathbb{R}^{m}$ is given by $f_{G}(\mathbf{p})=\left(\left\|e_{1}\right\|^{2},\left\|e_{2}\right\|^{2}, \ldots,\left\|e_{m}\right\|^{2}\right)$, where $\left\|e_{i}\right\|=\|p(u)-p(v)\|$, when $e_{i}=u v$. Note that the evaluation of the Jacobian of the rigidity map at the point $\mathbf{p} \in \mathbb{R}^{2 n},\left.d f_{G}\right|_{\mathbf{p}}$, is twice the rigidity matrix of the framework $(G, p)$. 
Lemma 3.3. If $(G, p)$ is a quasi-generic framework and $G$ is $M$-independent then $f_{G}(p)$ is generic.

Proof. Choose a generic framework $(G, q)$ conguent to $(G, p)$. Since $G$ is $M$-independent, rank $\left.d f_{G}\right|_{\mathbf{q}}=|E|$. Hence Lemma 3.1 implies that $f_{G}(\mathbf{q})$ is generic. The lemma now follows since $f_{G}(\mathbf{p})=f_{G}(\mathbf{q})$.

Lemma 3.4. Suppose that $(G, p)$ is in standard position, $G$ is minimally rigid and $f_{G}(p)$ is generic. Let $\mathbf{p}=\left(0,0,0, y_{2}, x_{3}, y_{3}, \ldots, x_{n}, y_{n}\right), L=\mathbb{Q}(\mathbf{p})$ and $K=\mathbb{Q}\left(f_{G}(\mathbf{p})\right)$. Then $\left(y_{2}, x_{3}, y_{3}, \ldots, x_{n}, y_{n}\right)$ is generic and $\tilde{K}=\tilde{L}$.

Proof. Define $f: \mathbb{R}^{2 n-3} \rightarrow \mathbb{R}^{2 n-3}$ by

$$
f\left(z_{1}, z_{2}, \ldots, z_{2 n-3}\right)=f_{G}\left(0,0,0, z_{1}, z_{2}, \ldots, z_{2 n-3}\right) .
$$

Let $\mathbf{p}^{\prime}=\left(y_{2}, x_{3}, y_{3}, \ldots, x_{n}, y_{n}\right)$. Then $f\left(\mathbf{p}^{\prime}\right)=f_{G}(\mathbf{p})$ is generic. We have $L=\mathbb{Q}\left(\mathbf{p}^{\prime}\right)$ and $K=\mathbb{Q}\left(f\left(\mathbf{p}^{\prime}\right)\right)$. By Lemma 3.2, we have $\tilde{K}=\tilde{L}$. Furthermore, $2 n-3=t d[\tilde{K}, \mathbb{Q}]=$ $t d[\tilde{L}, \mathbb{Q}]$. Thus $\mathbf{p}^{\prime}$ is a generic point in $\mathbb{R}^{2 n-3}$.

Lemma 3.5. Let $C=\left\{p_{1}, p_{2}, \ldots, p_{n}\right\}$ be a configuration. Then $C$ is quasi-generic if and only if there is an isometry $T$ of $\mathbb{R}^{2}$ such that $T\left(p_{1}\right)=(0,0), T\left(p_{2}\right)=\left(0, y_{2}\right)$, $T\left(p_{i}\right)=\left(x_{i}, y_{i}\right)$ for $3 \leq i \leq n$, and $\left\{y_{2}, x_{3}, y_{3}, \ldots, x_{n}, y_{n}\right\}$ is algebraically independent over $\mathbb{Q}$.

Proof. Suppose $C$ is quasi-generic. Let $G=(V, E)$ be a minimally rigid graph, $V=\left\{v_{1}, v_{2}, \ldots, v_{n}\right\}$, and define $p: V \rightarrow \mathbb{R}^{2}$ by $p\left(v_{i}\right)=p_{i}$ for $1 \leq i \leq n$. Consider the quasi-generic framework $(G, p)$. By Lemma $3.3, f_{G}(\mathbf{p})$ is a generic point in $\mathbb{R}^{2 n-3}$. Choose an isometry $T$ of $\mathbb{R}^{2}$ which maps $(G, p)$ to a framework $(G, q)$ such that $T\left(p_{1}\right)=(0,0), T\left(p_{2}\right)=\left(0, y_{2}\right)$ and $T\left(p_{i}\right)=\left(x_{i}, y_{i}\right)$ for $3 \leq i \leq$ $n$. Then $q=\left(0,0,0, y_{2}, x_{3}, y_{3}, \ldots, x_{n}, y_{n}\right)$ and $f_{G}(q)=f_{G}(p)$. By Lemma 3.4, $\left\{y_{2}, x_{3}, y_{3}, \ldots, x_{n}, y_{n}\right\}$ is algebraically independent over $\mathbb{Q}$.

We next suppose that there is an isometry of $\mathbb{R}^{2}$ which maps $C$ onto $C^{\prime}=\{(0,0)$, $\left.\left(0, y_{2}\right),\left(x_{3}, y_{3}\right), \ldots,\left(x_{n}, y_{n}\right)\right\}$ and $\left\{y_{2}, x_{3}, y_{3}, \ldots, x_{n}, y_{n}\right\}$ is algebraically independent over $\mathbb{Q}$. Choose $\theta \in \mathbb{R}$ such that $\left\{\sin \theta, y_{2}, x_{3}, y_{3}, \ldots, x_{n}, y_{n}\right\}$ is algebraically independent over $\mathbb{Q}$. Let $T_{1}$ be the isometry of $\mathbb{R}^{2}$ which rotates the plane through $\theta$ radians about the origin. Let $T_{1}\left(C^{\prime}\right)=C_{1}$. Then $C_{1}=\left\{(0,0),\left(s_{2}, t_{2}\right),\left(s_{3}, t_{3}\right), \ldots,\left(s_{n}, t_{n}\right)\right\}$ where $\left(s_{2}, t_{2}\right)=\left(-y_{2} \sin \theta, y_{2} \cos \theta\right)$ and $\left(s_{i}, t_{i}\right)=\left(x_{i} \cos \theta-y_{i} \sin \theta, x_{i} \sin \theta+y_{i} \cos \theta\right)$ for $3 \leq i \leq n$.

Claim 3.6. $\left\{s_{2}, t_{2}, s_{3}, t_{3}, \ldots, s_{n}, t_{n}\right\}$ is algebraically independent over $\mathbb{Q}$.

Proof. $\quad$ Let $K=\mathbb{Q}\left(\sin \theta, y_{2}, x_{3}, y_{3}, \ldots, x_{n}, y_{n}\right)$ and $L=\mathbb{Q}\left(s_{2}, t_{2}, s_{3}, t_{3}, \ldots, s_{n}, t_{n}\right)$. We show that $\tilde{K} \subseteq \tilde{L}$. It suffices to show that $\sin \theta, y_{2}, x_{3}, y_{3}, \ldots, x_{n}, y_{n}$ are all algebraic over $L$. We have $y_{2}^{2}=s_{2}^{2}+t_{2}^{2} \in L$ so $y_{2} \in \tilde{L}$. Thus $\sin \theta=-s_{2} / y_{2} \in \tilde{L}$ and $\cos \theta=$ 
$t_{2} / y_{2} \in \tilde{L}$. Let $\ell_{1}=\sin \theta$ and $\ell_{2}=\cos \theta$. For each $3 \leq i \leq n$, we have $s_{i}=\ell_{1} x_{i}-\ell_{2} y_{i}$ and $t_{i}=\ell_{2} x_{i}+\ell_{1} y_{i}$. We can solve these equations to express $x_{i}, y_{i}$ as rational functions of $s_{i}, t_{i}, \ell_{1}, \ell_{2}$. Thus $x_{i}, y_{i} \in \tilde{L}$. Hence $\tilde{K} \subseteq \tilde{L}$ and $t d[L: \mathbb{Q}] \geq t d[K: \mathbb{Q}]=2 n-2$. Thus $\left\{s_{2}, t_{2}, s_{3}, t_{3}, \ldots, s_{n}, t_{n}\right\}$ is algebraically independent over $\mathbb{Q}$.

Choose $\beta, \gamma \in \mathbb{R}$ such that $\left\{\beta, \gamma, s_{2}, t_{2}, s_{3}, t_{3}, \ldots, s_{n}, t_{n}\right\}$ is algebraically independent over $\mathbb{Q}$. Let $T_{2}$ be the isometry of $\mathbb{R}^{2}$ which translates the plane by $(\beta, \gamma)$. Let $T_{2}\left(C_{1}\right)=C_{2}$. Then $C_{2}=\left\{\left(w_{1}, z_{1}\right),\left(w_{2}, z_{2}\right), \ldots,\left(w_{n}, z_{n}\right)\right\}$ where $\left(w_{1}, z_{1}\right)=(\beta, \gamma)$ and $\left(w_{i}, z_{i}\right)=\left(s_{i}+\beta, t_{i}+\gamma\right)$ for $2 \leq i \leq n$. It can easily be seen that $\mathbb{Q}\left(\beta, \gamma, s_{2}, t_{2}, s_{3}\right.$, $\left.t_{3}, \ldots, s_{n}, t_{n}\right)=\mathbb{Q}\left(w_{1}, z_{1}, w_{2}, z_{2}, \ldots, w_{n}, z_{n}\right)$. Hence $\left\{w_{1}, z_{1}, w_{2}, z_{2}, \ldots, w_{n}, z_{n}\right\}$ is algebraically independent over $\mathbb{Q}$. Thus $C^{\prime}$ is congruent to the generic configuration $C_{2}$. Since $C$ is congruent to $C^{\prime}$, it follows that $C$ is quasi-generic.

Corollary 3.7. Suppose that $(G, p)$ is a rigid generic framework and that $(G, q)$ is equivalent to $(G, p)$. Then $(G, q)$ is quasi-generic.

Proof. Let $H$ be a minimally rigid spanning subgraph of $G$. Choose isometries of $\mathbb{R}^{2}$ which map $(H, p)$ and $(H, q)$ to two frameworks $\left(H, p^{\prime}\right)$ and $\left(H, q^{\prime}\right)$ in standard position. By Lemma 3.3, $f_{H}(p)$ is generic. Thus $f_{H}\left(q^{\prime}\right)=f_{H}\left(p^{\prime}\right)=f_{H}(p)$ is generic. By Lemmas 3.4 and 3.5, $\left(H, q^{\prime}\right)$ is quasi-generic. Hence $(H, q)$ and $(G, q)$ are quasigeneric.

\section{1-Extensions and Globally Linked Pairs}

Let $(G, p)$ be a framework and $u, v \in V$. Recall that $\{u, v\}$ is globally linked in $(G, p)$ if, in all equivalent frameworks $(G, q)$, we have $\|p(u)-p(v)\|=\|q(u)-q(v)\|$. The pair $\{u, v\}$ is globally linked in $G$ if it is globally linked in all generic frameworks $(G, p)$. Note that Corollary 3.7 implies that a pair of vertices $\{u, v\}$ in a rigid graph $G$ is globally linked if and only if we have $\|p(u)-p(v)\|=\|q(u)-q(v)\|$ for all equivalent pairs of quasi-generic frameworks $(G, p)$ and $(G, q)$. For $v \in V(G)$ let $N_{G}(v)$ denote the set of vertices adjacent to vertex $v$ in graph $G$.

Lemma 4.1. Let $G$ be a graph, and $v \in V(G)$ with $N_{G}(v)=\{u, w$, t $\}$. If $G-v$ is rigid then $\{u, w\}$ is globally linked in $G$.

Proof. Let $\left(G, p^{*}\right)$ and $\left(G, q^{*}\right)$ be equivalent quasi-generic frameworks. By Lemma $3.5,\left(G, p^{*}\right)$ is congruent to a framework $(G, p)$, where $p=\left(0,0,0, p_{4}, p_{5}, \ldots, p_{2 n}\right)$, $p(u)=(0,0), p(w)=\left(0, p_{4}\right), p(t)=\left(p_{5}, p_{6}\right), p(v)=\left(p_{2 n-1}, p_{2 n}\right)$ and $\left\{p_{4}, p_{5}, \ldots, p_{2 n}\right\}$ is algebraically independent over $\mathbb{Q}$. Similarly $\left(G, q^{*}\right)$ is congruent to a framework $(G, q)$, where $q(u)=(0,0), q(w)=\left(0, q_{4}\right), q(t)=\left(q_{5}, q_{6}\right)$ and $q(v)=\left(q_{2 n-1}, q_{2 n}\right)$. Then

$\left\|p^{*}(u)-p^{*}(w)\right\|^{2}-\left\|q^{*}(u)-q^{*}(w)\right\|^{2}=\|p(u)-p(w)\|^{2}-\|q(u)-q(w)\|^{2}=p_{4}^{2}-q_{4}^{2}$.

Hence it will suffice to show that $p_{4}^{2}-q_{4}^{2}=0$. By symmetry we may suppose that $p_{4}^{2}-q_{4}^{2} \geq 0$. 
Let $p^{\prime}=\left.p\right|_{V-v}$ and $q^{\prime}=\left.q\right|_{V-v}$. Consider the equivalent quasi-generic frameworks $\left(G-v, p^{\prime}\right)$ and $\left(G-v, q^{\prime}\right)$. Applying Lemmas 3.3 and 3.4 to a minimally rigid spanning subgraph of $G-v$, we have $\tilde{K}=\tilde{L}$ where $K=\mathbb{Q}\left(p^{\prime}\right)$ and $L=\mathbb{Q}\left(q^{\prime}\right)$. Thus $q_{4}, q_{5}, q_{6} \in$ $\tilde{K}$. Since $(G, q)$ is equivalent to $(G, p)$, we have the following equations:

$$
\begin{aligned}
q_{2 n-1}^{2}+q_{2 n}^{2} & =p_{2 n-1}^{2}+p_{2 n}^{2}, \\
q_{2 n-1}^{2}+\left(q_{2 n}-q_{4}\right)^{2} & =p_{2 n-1}^{2}+\left(p_{2 n}-p_{4}\right)^{2}, \\
\left(q_{2 n-1}-q_{5}\right)^{2}+\left(q_{2 n}-q_{6}\right)^{2} & =\left(p_{2 n-1}-p_{5}\right)^{2}+\left(p_{2 n}-p_{6}\right)^{2} .
\end{aligned}
$$

Using (1) and (2) and the fact that $q^{\prime}$ is generic (and hence $q_{4} \neq 0$ ) we get that

$$
q_{2 n}=\frac{q_{4}^{2}-p_{4}^{2}+2 p_{2 n} p_{4}}{2 q_{4}} .
$$

Similarly, using (1), (3) and (4) we get that

$$
q_{2 n-1}=\frac{q_{5}^{2}+q_{6}^{2}-p_{5}^{2}-p_{6}^{2}+2 p_{2 n-1} p_{5}+2 p_{2 n} p_{6}-q_{6}\left(\left(q_{4}^{2}-p_{4}^{2}+2 p_{2 n} p_{4}\right) / q_{4}\right)}{2 q_{5}} .
$$

From (1) we know that $4 q_{4}^{2} q_{5}^{2}\left(q_{2 n-1}^{2}+q_{2 n}^{2}-p_{2 n-1}^{2}-p_{2 n}^{2}\right)=0$. Using (4) and (5) to substitute for $q_{2 n-1}$ and $q_{2 n}$, we obtain

$$
a_{11} p_{2 n-1}^{2}+a_{22} p_{2 n}^{2}+a_{12} p_{2 n-1} p_{2 n}+a_{1} p_{2 n-1}+a_{2} p_{2 n}+a_{0}=0
$$

where $a_{11}, a_{22}, a_{12}, a_{1}, a_{2}, a_{0} \in \tilde{K}$. This means that there is a polynomial

$$
f=a_{11} z_{1}^{2}+a_{22} z_{2}^{2}+a_{12} z_{1} z_{2}+a_{1} z_{1}+a_{2} z_{2}+a_{0} \in \tilde{K}\left[z_{1}, z_{2}\right]
$$

such that $f\left(p_{2 n-1}, p_{2 n}\right)=0$. Since $\left\{p_{4}, p_{5}, \ldots, p_{2 n}\right\}$ is algebraically independent over $\mathbb{Q},\left\{p_{2 n-1}, p_{2 n}\right\}$ is algebraically independent over $\tilde{K}$. Thus $f \equiv 0$. In particular,

$$
a_{22}=4 q_{5}^{2}\left(p_{4}^{2}-q_{4}^{2}\right)+4\left(p_{4} q_{6}-q_{4} p_{6}\right)^{2}=0 .
$$

Since $p_{4}^{2}-q_{4}^{2} \geq 0$ we must have $p_{4}^{2}-q_{4}^{2}=0$.

Theorem 4.2. Let $G=(V, E)$ be a graph, $x, y, v \in V, N_{G}(v)=\{u, w, t\}, u w \notin E$ and $H=G-v+u w$. Suppose that $H-u w$ is rigid and that $\{x, y\}$ is globally linked in $H$. Then $\{x, y\}$ is globally linked in $G$.

Proof. Suppose $(G, p)$ is a generic framework and that $(G, q)$ is equivalent to $(G, p)$. Let $p^{\prime}=\left.p\right|_{V-v}$ and $q^{\prime}=\left.q\right|_{V-v}$. Since $G-v=H-u w$ is rigid, Lemma 4.1 implies that $\{u, w\}$ is globally linked in $G$. Thus

$$
\left\|p^{\prime}(u)-p^{\prime}(w)\right\|=\|p(u)-p(w)\|=\|q(u)-q(w)\|=\left\|q^{\prime}(u)-q^{\prime}(w)\right\| .
$$

Hence $\left(H, p^{\prime}\right)$ and $\left(H, q^{\prime}\right)$ are equivalent. Since $\{x, y\}$ is globally linked in $H$, we have

$$
\|p(x)-p(y)\|=\left\|p^{\prime}(x)-p^{\prime}(y)\right\|=\left\|q^{\prime}(x)-q^{\prime}(y)\right\|=\|q(x)-q(y)\| .
$$

Thus $\{x, y\}$ is globally linked in $G$. 
Corollary 4.3. Suppose that $H$ is globally rigid with $|V(H)| \geq 4$ and $G$ is obtained from $H$ by a 1-extension. Then $G$ is globally rigid.

Proof. Let $H=G-v+u w$. Since $H$ is globally rigid, $H-e$ is rigid for all edges $e$ of $H$ by Theorem 1.1. Hence $H-u w$ is rigid. Theorem 4.2 and the fact that $H$ is globally rigid now imply that all pairs $\{x, y\} \subseteq V-v$ are globally linked in $G$. Suppose $(G, p)$ is a generic framework and that $(G, q)$ is equivalent to $(G, p)$. Let $p^{\prime}=\left.p\right|_{V-v}$ and $q^{\prime}=\left.q\right|_{V-v}$. Since all pairs $\{x, y\} \subseteq V-v$ are globally linked in $G,\left(G-v, p^{\prime}\right)$ is congruent to $\left(G-v, q^{\prime}\right)$. Since $(G, p)$ is generic and $v$ has three neighbours in $G$, this congruence extends to a congruence between $(G, p)$ and $(G, q)$.

Corollary 4.3 immediately implies Theorem 1.2, which, as mentioned in the Introduction, is a key step in the characterization of globally rigid graphs.

We close this section with some remarks on the $d$-dimensional case. Connelly's results in [6] imply that Theorem 1.2 can be extended to $d$-dimensions as follows. Given a graph $G=(V, E)$ and distinct vertices $x_{1}, x_{2}, \ldots, x_{d+1} \in V$ with $x_{1} x_{2} \in E, \mathrm{a}(1, d)$-extension of $G$ is a graph obtained from $G$ by deleting the edge $x_{1} x_{2}$ and adding a new vertex $z$ and new edges $z x_{1}, z x_{2}, \ldots, z x_{d+1}$.

Theorem 4.4 [6]. Let $G$ be a graph and let $d$ be a positive integer. Suppose that $G$ can be obtained from $K_{d+2}$ by a sequence of $(1, d)$-extensions and edge additions. Then every generic realization of $G$ in $\mathbb{R}^{d}$ is globally rigid.

We do not know if Lemma 4.1, Theorem 4.2 and Corollary 4.3 can be extended to $d$-dimensions.

\section{Globally Linked Pairs in $M$-Connected Graphs}

Given a graph $G=(V, E)$, a subgraph $H=(W, C)$ is said to be an $M$-circuit in $G$ if $C$ is a circuit (i.e. a minimal dependent set) in $\mathcal{R}(G)$. In particular, $G$ is an $M$-circuit if $E$ is a circuit in $\mathcal{R}(G)$. Using Theorem 2.2 we may deduce that $G$ is an $M$-circuit if and only if $|E|=2|V|-2$ and $G-e$ is minimally rigid for all $e \in E$. Recall that a graph $G$ is redundantly rigid if $G-e$ is rigid for all $e \in E$. Note also that a graph $G$ is redundantly rigid if and only if $G$ is rigid and each edge of $G$ belongs to a circuit in $\mathcal{R}(G)$, i.e. an $M$-circuit of $G$.

Any two maximal redundantly rigid subgraphs of a graph $G=(V, E)$ can have at most one vertex in common, and hence are edge-disjoint (see [13]). Defining a redundantly rigid component of $G$ to be either a maximal redundantly rigid subgraph of $G$, or a subgraph induced by an edge which belongs to no $M$-circuit of $G$, we deduce that the redundantly rigid components of $G$ partition $E$. Since each redundantly rigid component is rigid, this partition is a refinement of the partition of $E$ given by the rigid components of $G$. Note that the redundantly rigid components of $G$ are induced subgraphs of $G$.

Given a matroid $\mathcal{M}=(E, \mathcal{I})$, we define a relation on $E$ by saying that $e, f \in E$ are related if $e=f$ or if there is a circuit $C$ in $\mathcal{M}$ with $e, f \in C$. It is well known that this 
is an equivalence relation. The equivalence classes are called the components of $\mathcal{M}$. If $\mathcal{M}$ has at least two elements and only one component then $\mathcal{M}$ is said to be connected.

We say that a graph $G=(V, E)$ is $M$-connected if $\mathcal{R}(G)$ is connected. Thus $M$ circuits are special $M$-connected graphs. Another example is the complete bipartite graph $K_{3, m}$, which is $M$-connected for all $m \geq 4$. The $M$-components of $G$ are the subgraphs of $G$ induced by the components of $\mathcal{R}(G)$. Note that the $M$-components of $G$ are induced subgraphs. For more examples and basic properties of $M$-circuits and $M$-connected graphs see [1] and [13]. In this paper we shall need the following lemmas.

We say that a graph $G$ is nearly 3-connected if $G$ can be made 3-connected by adding at most one new edge. We need the following result on $M$-connected graphs. The first part appears as Lemma 3.1 of [13]. The second part was proved in Theorem 3.2 of [13] for redundantly rigid graphs. The same proof goes through under the weaker hypothesis that each edge of $G$ is in an $M$-circuit.

Theorem 5.1 [13].

(a) If $G$ is $M$-connected then $G$ is redundantly rigid.

(b) If $G$ is nearly 3-connected and each edge of $G$ is in an $M$-circuit then $G$ is M-connected.

Note that Theorems 1.4 and 5.1 imply that a graph with at least four vertices is globally rigid if and only if it is 3-connected and $M$-connected.

Given two graphs $H_{1}=\left(V_{1}, E_{1}\right)$ and $H_{2}=\left(V_{2}, E_{2}\right)$ with $V_{1} \cap V_{2}=\emptyset$ and two designated edges $u_{1} v_{1} \in E_{1}$ and $u_{2} v_{2} \in E_{2}$, the 2-sum of $H_{1}$ and $H_{2}$ (along the edge pair $u_{1} v_{1}, u_{2} v_{2}$ ) is the graph obtained from $H_{1}-u_{1} v_{1}$ and $H_{2}-u_{2} v_{2}$ by identifying $u_{1}$ with $u_{2}$ and $v_{1}$ with $v_{2}$, see Fig. 3. We denote a 2-sum of $H_{1}$ and $H_{2}$ by $H_{1} \oplus_{2} H_{2}$.

Lemma 5.2. Suppose $G_{1}$ and $G_{2}$ are graphs and $G=G_{1} \oplus_{2} G_{2}$.

(a) [1, Lemma 4.1] If $G_{1}$ and $G_{2}$ are $M$-circuits then $G$ is an $M$-circuit.

(b) [13, Lemma 3.3] If $G_{1}$ and $G_{2}$ are M-connected then $G$ is $M$-connected.

A $j$-separation of a graph $H=(V, E)$ is a pair $\left(H_{1}, H_{2}\right)$ of edge-disjoint subgraphs of $H$ each with at least $j+1$ vertices such that $H=H_{1} \cup H_{2}$ and $\left|V\left(H_{1}\right) \cap V\left(H_{2}\right)\right|=j$. Note that $H$ is 3-connected if and only if $H$ has at least four vertices and has no $j$ separation for all $0 \leq j \leq 2$. If $\left(H_{1}, H_{2}\right)$ is a 2-separation of $H$, then we say that $V\left(H_{1}\right) \cap V\left(H_{2}\right)$ is a 2-separator of $H$.

Let $G=(V, E)$ be a 2-connected graph and suppose that $\left(H_{1}, H_{2}\right)$ is a 2-separation of $G$ with $V\left(H_{1}\right) \cap V\left(H_{2}\right)=\{u, v\}$. For $1 \leq i \leq 2$, let $H_{i}^{\prime}=H_{i}+u v$ if $u v \notin E\left(H_{i}\right)$ and otherwise put $H_{i}^{\prime}=H_{i}$. We say that $H_{1}^{\prime}, H_{2}^{\prime}$ are the cleavage graphs obtained by cleaving $G$ along $\{u, v\}$.

Lemma 5.3. Suppose $G$ is a 2-connected graph and $G_{1}$ and $G_{2}$ are cleavage graphs obtained by cleaving $G$ along a 2-separator $\{u, v\}$.

(a) [1, Lemmas 2.4(c), 4.2] If $G$ is an $M$-circuit then $u v \notin E(G)$, and $G_{1}$ and $G_{2}$ are both $M$-circuits.

(b) [13, Lemma 3.4] If $G$ is $M$-connected then $G_{1}$ and $G_{2}$ are also $M$-connected. 


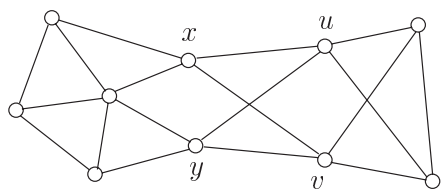

Fig. 3. An $M$-circuit $G$ obtained from a 'wheel' on six vertices and two copies of $K_{4}$ by taking 2 -sums. The identified pairs of vertices, $\{u, v\}$ and $\{x, y\}$, are globally linked in $G$.

We can use Theorem 4.2 to characterize globally linked pairs in $M$-connected graphs. First we need some preliminary lemmas, illustrated by Fig. 3 .

Lemma 5.4. Let $G_{1}, G_{2}$ be $M$-circuits such that $G_{1}$ is 3-connected. Let $G=G_{1} \oplus_{2} G_{2}$, where the pair of identified vertices is $\{x, y\}$. Then $\{x, y\}$ is globally linked in $G$.

Proof. We use induction on $\left|V\left(G_{1}\right)\right|$. Suppose that the 2-sum was obtained along the edges $x_{i}, y_{i} \in E\left(G_{i}\right), 1 \leq i \leq 2$. If $G_{1}=K_{4}$, with $V\left(G_{1}\right)=\left\{v, t, x_{1}, y_{1}\right\}$, then $G-v=G_{2}-x_{2} y_{2}+t+\left\{t x_{2}, t y_{2}\right\}$. Since $G_{2}$ is redundantly rigid by Theorem 5.1(a), $G_{2}-x_{2} y_{2}$, and hence also $G-v$, are rigid. By Lemma $4.1,\{x, y\}$ is globally linked in $G$. Thus we may suppose that $\left|V\left(G_{1}\right)\right| \geq 5$.

By Theorem 5.9 of [1] there is $v \in V\left(G_{1}\right)-\left\{x_{1}, y_{1}\right\}$, with $N(v)=\{u, w, t\}$, such that $G_{1}^{v}=G-v+u w$ is a 3-connected $M$-circuit. Let $H=G_{1}^{v} \oplus_{2} G_{2}$ be the 2-sum along the edge pair $x_{1} y_{1}, x_{2} y_{2}$. Then $H$ is an $M$-circuit by Lemma 5.2(a), and hence, by induction, $\{x, y\}$ is globally linked in $H$. Since $H$ is an $M$-circuit, $H-u w$ is rigid. Hence by Theorem 4.2, $\{x, y\}$ is globally linked in $G$.

Corollary 5.5. Let $G$ be an $M$-circuit and let $\{u, v\}$ be a 2-separator of $G$. Then $\{u, v\}$ is globally linked in $G$.

Proof. We use induction on $|V(G)|$. Since $G$ is an $M$-circuit and is not 3-connected, we can choose a 2-separator $\{x, y\}$ in $G$ and express $G$ as $G=G_{1} \oplus_{2} G_{2}$, where the pair of identified vertices is $\{x, y\}$. Suppose that this 2-sum was obtained along the edges $x_{i}, y_{i} \in E\left(G_{i}\right), 1 \leq i \leq 2$. By Lemma 5.3(a), $x y \notin E(G)$ and $G_{1}, G_{2}$ are $M$-circuits. By choosing $\{x, y\}$ so that $G_{1}$ is minimal, we may also ensure that $G_{1}$ is 3-connected. By Lemma 5.4, $\{x, y\}$ is globally linked in $G$. Thus we may suppose that $\{u, v\} \neq\{x, y\}$. Since $G_{1}$ is 3 -connected, $\{u, v\}$ is a 2 -separator of $G_{2}$. By induction, $\{u, v\}$ is globally linked in $G_{2}$. Since $\{x, y\}$ is globally linked in $G$ and $\left(G_{2}-x_{2} y_{2}\right) \subseteq G$, it follows that $\{u, v\}$ is also globally linked in $G$.

Let $H=(V, E)$ be a graph and $x, y \in V$. We use $\kappa_{H}(x, y)$ to denote the maximum number of pairwise openly disjoint $x y$-paths in $H$. If $x y \notin E$ then, by Menger's theorem, $\kappa_{H}(x, y)$ is equal to the size of a smallest set $S \subseteq V(H)-\{x, y\}$ for which there is no $x y$-path in $H-S$.

Lemma 5.6. Let $(G, p)$ be a generic framework, $x, y \in V(G), x y \notin E(G)$, and suppose that $\kappa_{G}(x, y) \leq 2$. Then $\{x, y\}$ is not globally linked in $(G, p)$. 
Proof. Since there do not exist three pairwise openly disjoint $x y$-paths in $G$, it follows from Menger's theorem that there exists $u, v \in V(G)$ such that $x$ and $y$ belong to different components of $G-\{u, v\}$. Let $H$ be the component of $G-\{u, v\}$ which contains $x$. Construct $(G, q)$ from $(G, p)$ by reflecting $p(V(H))$ in the line through $p(u), p(v)$. Then $(G, p)$ is equivalent to $(G, q)$. Furthermore, $\|p(x)-p(y)\| \neq\|q(x)-q(y)\|$, since $p(y)=q(y)$ and, since $(G, p)$ is generic, $p(y)$ does not lie on the line through $p(u), p(v)$. Thus $\{x, y\}$ is not globally linked in $(G, p)$.

Theorem 5.7. Let $G=(V, E)$ be an $M$-connected graph and $x, y \in V$. Then $\{x, y\}$ is globally linked in $G$ if and only if $\kappa_{G}(x, y) \geq 3$.

Proof. We first prove necessity. Suppose that $\{x, y\}$ is globally linked. If $x y \notin E$ then the existence of three openly disjoint $x y$-paths follows from Lemma 5.6. If $x y \in E$ then, since $G$ is $M$-connected, $G-x y$ is rigid by Theorem 5.1(a). Since rigid graphs are 2-connected, we have two openly disjoint $x y$-paths in $G-x y$. Thus we have three openly disjoint $x y$-paths in $G$.

We next prove sufficiency. Suppose that there exist three pairwise openly disjoint $x y$-paths in $G$. We use induction on $|V(G)|$ to show that $\{x, y\}$ is globally linked in $G$. If $G$ is 3-connected then $G$ is globally rigid by Theorems 1.4 and 5.1(a), and hence $\{x, y\}$ is globally linked in $G$. Thus we may suppose that $G-\{u, v\}$ is disconnected for some $u, v \in V$. Choose two vertices $w, z$ belonging to different components of $G-\{u, v\}$. Since $G$ is $M$-connected, there exists an $M$-circuit $H$ in $G$ with $w, x \in V(H)$. Then $\{u, v\}$ is a 2-separator of $H$. By Corollary 5.5, $\{u, v\}$ is globally linked in $H$. Thus $\{u, v\}$ is globally linked in $G$.

Let $G_{1}, G_{2}$ be the cleavage graphs obtained by cleaving $G$ along the 2 -separator $\{u, v\}$. The graphs $G_{1}, G_{2}$ are both $M$-connected by Lemma 5.3(b). Using the fact that there are three pairwise openly disjoint $x y$-paths in $G$, and relabelling if necessary, we have $x, y \in V\left(G_{1}\right)$. It is easy to see that there are three pairwise openly disjoint $x y$-paths in $G_{1}$. By induction $\{x, y\}$ is globally linked in $G_{1}$. Since $\{u, v\}$ is globally linked in $G$ and $\left(G_{1}-u_{1} v_{1}\right) \subseteq G,\{x, y\}$ is also globally linked in $G$.

Theorem 5.7 has the following immediate corollary.

Corollary 5.8. Let $G=(V, E)$ be a graph and $x, y \in V$. If either $x y \in E$, or there is an $M$-component $H$ of $G$ with $\{x, y\} \subseteq V(H)$ and $\kappa_{H}(x, y) \geq 3$, then $\{x, y\}$ is globally linked in $G$.

We conjecture that the converse is also true.

Conjecture 5.9. The pair $\{x, y\}$ is globally linked in a graph $G=(V, E)$ if and only if either $x y \in E$ or there is an M-component $H$ of $G$ with $\{x, y\} \subseteq V(H)$ and $\kappa_{H}(x, y) \geq 3$.

We shall verify Conjecture 5.9 for minimally rigid graphs that can be obtained from an edge by iteratively adding vertices of degree 2 . The Henneberg 0 -extension operation on vertices $x, y$ in a graph $G$ adds a new vertex $z$ and new edges $x z, y z$ to $G$. 
Lemma 5.10. If $\{u, v\}$ is not globally linked in $H$ and $G$ is a 0-extension of $H$ then $\{u, v\}$ is not globally linked in $G$.

Proof. Since $\{u, v\}$ is not globally linked in $H$, there exists a generic framework $(H, p)$, and an equivalent framework $(H, q)$, such that $\|p(u)-p(v)\| \neq\|q(u)-q(v)\|$. Let $G$ be obtained from $H$ by adding vertex $w$ and edges $w x, w y$. Let $\alpha_{1}, \alpha_{2}$ be two real numbers such that the set containing $\alpha_{1}, \alpha_{2}$, and the entries in $f_{H}(p)$ is algebraically independent over $\mathbb{Q}$, and such that $\alpha_{1}+\alpha_{2}$ is large enough and $\alpha_{1}-\alpha_{2}$ is small enough. (Note that $f_{H}(p)$ is generic by Lemma 3.3.) Now we may choose a pair of points $r_{p}, r_{q}$ in $\mathbb{R}^{2}$ such that $\left\|r_{p}-p(x)\right\|^{2}=\alpha_{1}=\left\|r_{q}-q(x)\right\|^{2}$ and $\left\|r_{p}-p(y)\right\|^{2}=\alpha_{2}=\left\|r_{q}-q(y)\right\|^{2}$. Thus extending $(H, p)$ by $p(w)=r_{p}$ and $(H, q)$ by $q(w)=r_{q}$ gives a pair of equivalent frameworks on $G$ such that $\|p(u)-p(v)\| \neq\|q(u)-q(v)\|$ holds. Note that (the extended) $p$ is quasi-generic by Lemmas 3.4 and 3.5.

Henneberg [12] showed that every minimally rigid graph can be obtained from $K_{2}$ by recursively performing 0 -extensions and 1-extensions. We say that $G$ is 2-simple if $G$ can be obtained from $K_{2}$ by recursively performing just 0 -extensions. For example, the graph of Fig. 1 is 2-simple. Note that all $M$-components (and all redundantly rigid components) in a minimally rigid graph are isomorphic to $K_{2}$. Thus to prove Conjecture 5.9 for minimally rigid graphs $G$ we have to show that the only globally linked pairs in $G$ are the pairs of adjacent vertices.

Theorem 5.11. Let $G=(V, E)$ be a 2-simple graph and suppose that $u v \notin E$. Then $\{u, v\}$ is not globally linked.

Proof. The proof is by induction on $|V|$. The theorem is trivially true for $|V| \leq 3$, so we may assume that $|V| \geq 4$ and that the theorem holds for all 2-simple graphs with at most $|V|-1$ vertices. Since $G$ is 2 -simple, it has a vertex $w$ of degree 2. If $w \in\{u, v\}$ then $\kappa_{G}(u, v)=2$ and hence $\{u, v\}$ is not globally linked by Lemma 5.6. So suppose $w \neq u, v$ and consider $H=G-w . H$ is also 2-simple and $u v \notin E(H)$. By induction this implies that $\{u, v\}$ is not globally linked in $H$. Since $G$ is a 0 -extension of $H$, the theorem follows from Lemma 5.10.

In order to extend Theorem 5.11 to all minimally rigid graphs, it would suffice to find an analogous result to Lemma 5.10 for 1-extensions.

We have attempted to prove Conjecture 5.9 by considering two other conjectures on globally linked pairs which together are equivalent to Conjecture 5.9.

Conjecture 5.12. Suppose that $\{x, y\}$ is a globally linked pair in a graph $G$. Then there is a redundantly rigid component $R$ of $G$ with $\{x, y\} \subseteq V(R)$.

Conjecture 5.13. Let $G$ be a graph. Suppose that there is a redundantly rigid component $R$ of $G$ with $\{x, y\} \subseteq V(R)$ and $\{x, y\}$ is globally linked in $G$. Then $\{x, y\}$ is globally linked in $R$. 
It follows from Theorem 5.1(a) that Conjecture 5.9 implies both Conjectures 5.12 and 5.13 .

The "if" direction of Conjecture 5.9 follows from Corollary 5.8. We prove that the "only if" direction follows from Conjectures 5.12 and 5.13.

Proof (of the "only if" part of Conjecture 5.9 by assuming Conjectures 5.12 and 5.13 are true). Suppose that $\{x, y\}$ is globally linked in $G=(V, E)$. We use induction on $|V|$ to show that either $x y \in E$ or there is an $M$-component $H$ of $G$ with $\{x, y\} \subseteq V(H)$ and $\kappa_{H}(x, y) \geq 3$. Since the statement is trivially true if $|V| \leq 3$, we may assume that $|V| \geq 4$ and that $x y \notin E$. It follows from the truth of Conjectures 5.12 and 5.13 that there is a redundantly rigid component $R$ of $G$ with $\{x, y\} \subseteq V(R)$ and such that $\{x, y\}$ is globally linked in $R$. This implies that $\kappa_{R}(x, y) \geq 3$ by Lemma 5.6. If $R$ is 3 -connected then $R$ is $M$-connected by Theorem 5.1(b), and we are done by choosing $H=R$.

Now suppose that there is a 2-separator $\{u, v\}$ of $R$ and let $R_{1}, R_{2}$ be the cleavage graphs obtained by cleaving $R$ along $\{u, v\}$. Since $\kappa_{R}(x, y) \geq 3$, we may assume, without loss of generality, that $x, y \in V\left(R_{1}\right)$. We also suppose that the 2-separator has been chosen so that $R_{2}$ is inclusionwise minimal. This implies that $R_{2}$ is 3-connected. (Note that $\left|V\left(R_{2}\right)\right| \geq 4$, since $R$ is redundantly rigid.)

Claim 5.14. There is an $M$-circuit $C$ in $R_{2}$ with $u v \in E(C)$.

Proof. Since $R$ is redundantly rigid, every edge $e \in E(R)$ belongs to an $M$-circuit $C_{e}$. Each $M$-circuit $C^{\prime}$ is a 2-connected subgraph of $R$. This fact and Lemma 5.3(a) imply that if $C_{e} \nsubseteq R_{2}$ for some $e \in E\left(R_{2}\right)-u v$, then the claim will follow by choosing $C=\left(C_{e} \cap R_{2}\right)+u v$. Thus we may suppose that $C_{e} \subset R_{2}-u v$ for all $e \in E\left(R_{2}\right)-u v$. Since $R_{2}$ is 3-connected, Theorem 5.1(b) implies that $R_{2}-u v$ is $M$-connected, and hence rigid. Thus there is an $M$-circuit $C$ in $R_{2}$ with $u v \in E(C)$.

Since $\{x, y\}$ is globally linked in $R,\{u, v\}$ is a 2-separation of $R$ and $u v \in E\left(R_{1}\right)$, it follows that $\{x, y\}$ is globally linked in $R_{1}$. By induction, there is an $M$-connected subgraph $H^{\prime}$ of $R_{1}$ with $x, y \in V\left(H^{\prime}\right)$ and $\kappa_{H^{\prime}}(x, y) \geq 3$. If $u v \notin E\left(H^{\prime}\right)$ then let $H$ be an $M$-component of $G$ containing $H^{\prime}$. Thus we may suppose that $u v \in E\left(H^{\prime}\right)$. By Lemma 5.2(b), $H^{\prime \prime}=H^{\prime} \oplus_{2} C$ is an $M$-connected subgraph of $G$ containing $x, y$ with $\kappa_{H^{\prime \prime}}(x, y) \geq 3$. The conjecture now follows by choosing an $M$-component $H$ of $G$ containing $H^{\prime \prime}$.

We close this section by noting that the $M$-components, and hence also the maximal globally rigid subgraphs, of a graph $G=(V, E)$ can be found in polynomial time, see [2] for details. Theorem 5.7 implies that one can identify even larger globally linked sets of vertices in $G$. A globally rigid cluster of $G$ is a maximal subset of $V$ in which all pairs of vertices are globally linked in $G$. By Corollary 5.8, the vertex sets of the "cleavage units" (see Section 3 of [13]) of the $M$-components of $G$ are globally linked sets in $G$. The truth of Conjecture 5.9 would imply that the vertex sets of these cleavage units are precisely the globally rigid clusters of $G$. For example, the maximal globally rigid subgraphs of the graph $G$ in Fig. 3 are the six copies of $K_{3}$ and the remaining four 
copies of $K_{2}$. On the other hand, $G$ has three cleavage units, the copy of the wheel on six vertices and the two copies of $K_{4}$. The globally rigid clusters of $G$ are precisely the vertex sets of these three cleavage units.

\section{Uniquely Localizable Vertices}

The theory of globally rigid graphs can be applied in localization problems of sensor networks, see for example [7]. In this section we consider another generalization of global rigidity, unique localizability, which also has direct applications in sensor network localization, see [9].

Let $(G, p)$ be a generic framework with a designated set $P \subseteq V(G)$ of vertices. We say that a vertex $v \in V(G)$ is uniquely localizable in $(G, p)$ with respect to $P$ if whenever $(G, q)$ is equivalent to $(G, p)$ and $p(b)=q(b)$ for all vertices $b \in P$, then we also have $p(v)=q(v)$. We can think of $P$ as the set of pinned vertices (or anchor nodes in a sensor network). Vertices in $P$ are clearly uniquely localizable. It is easy to observe that if $v \in V-P$ is uniquely localizable then $|P| \geq 3$ and there exist three openly disjoint paths from $v$ to $P$ (see Lemma 5.6). Note that unique localizablity is not a generic property. Consider the graph given in Figs. 1 and 2. If we pin the set $P=\{u, x, y\}$ in the framework of Fig. 1, then $v$ is uniquely localizable with respect to $P$. This is not the case if we pin the same set in Fig. 2. Thus the unique localizablity of $v$ with respect to $P$ depends on the lengths of the edges incident with $w$.

We call a vertex $v$ uniquely localizable in graph $G$, with respect to $P \subseteq V(G)$, if $v$ is uniquely localizable with respect to $P$ in all generic frameworks $(G, p)$. For a graph $G$ and a set $P \subseteq V(G)$ let $G+K(P)$ denote the graph obtained from $G$ by adding all edges $b b^{\prime}$ for which $b b^{\prime} \notin E$ and $b, b^{\prime} \in P$. The following lemma is easy to prove.

Lemma 6.1. Let $G=(V, E)$ be a graph, $P \subseteq V$ and $v \in V-P$. Then $v$ is uniquely localizable in $G$ with respect to $P$ if and only if $|P| \geq 3$ and $\{v, b\}$ is globally linked in $G+K(P)$ for all (or equivalently, for at least three) vertices $b \in P$.

Lemma 6.1 and Theorem 5.7 imply the following characterization of uniquely localizable vertices when $G+K(P)$ is $M$-connected.

Corollary 6.2. Let $G=(V, E)$ be a graph, $P \subseteq V$ and $v \in V-P$. Suppose that $G+K(P)$ is $M$-connected. Then $v$ is uniquely localizable in $G$ with respect to $P$ if and only if $|P| \geq 3$ and $\kappa(v, b) \geq 3$ for all $b \in P$.

Similarly, Lemma 6.1 and Conjecture 5.9 would imply the following characterization of uniquely localizable vertices in an arbitrary graph.

Conjecture 6.3. Let $G=(V, E)$ be a graph, $P \subseteq V$ and $v \in V-P$. Then $v$ is uniquely localizable in $G$ with respect to $P$ if and only if $|P| \geq 3$ and there is an $M$-component $H$ of $G+K(P)$ with $P+v \subseteq V(H)$ and $\kappa_{H}(v, b) \geq 3$ for all $b \in P$.

As noted in the previous section, the $M$-components of a graph can be found in polynomial time. More precisely, [2] gives an algorithm which determines the $M$-components 
of a graph $G=(V, E)$ in $O\left(|V|^{2}\right)$ time. We can also determine whether two vertices of $G$ are joined by three openly disjoint paths in $O(|V|+|E|)$ time, see [16].

\section{Globally Loose Pairs}

We say that a pair of vertices $\{u, v\}$ is globally loose in a graph $G$ if for every generic framework $(G, p)$ there exists an equivalent framework $(G, q)$ such that $\|p(u)-p(v)\| \neq$ $\|q(u)-q(v)\|$. It follows from Lemma 5.6 and Theorem 5.7 that if $G$ is $M$-connected then each pair $\{u, v\}$ is either globally linked or globally loose in $G$, and that $\{u, v\}$ is globally loose if and only if $\kappa_{G}(u, v)=2$. On the other hand, the pair $\{u, v\}$ in the rigid graph given in Fig. 1 is neither globally linked nor globally loose.

We shall obtain a sufficient condition for a pair $\{u, v\}$ to be globally loose in a graph $G$. An edge $e$ of a globally rigid graph $H$ is critical if $H-e$ is not globally rigid.

Theorem 7.1. Let $G=(V, E)$ be a graph and $u, v \in V$. Suppose that $u v \notin E$, and that $G$ has a globally rigid supergraph $H$ in which $u v$ is a critical edge. Then $\{u, v\}$ is globally loose in $G$.

Proof. Let $(G, p)$ be a generic framework and let $H$ be a globally rigid supergraph of $G$ in which $u v$ is critical. Since $u v$ is critical in $H$, it follows that $(H-u v, p)$ is not globally rigid. Thus there is an equivalent, but not congruent realization $(H-u v, q)$. Clearly, $\|p(u)-p(v)\| \neq\|q(u)-q(v)\|$ must hold. Now $G$ is a subgraph of $H-u v$, and hence the framework $(G, q)$ verifies that $\{u, v\}$ is globally loose in $G$.

We call a minimally rigid graph $G$ special if every proper rigid subgraph $H$ of $G$ is complete (and hence is a complete graph on two or three vertices). The graphs $K_{3,3}$ and the prism are both special, as well as all graphs which can be obtained from $K_{3,3}$ by the following operation: replace two incident edges $a b, b c$ by six edges $a a^{\prime}, a^{\prime} b, b c^{\prime}, c^{\prime} c, a c^{\prime}, a^{\prime} c$, where $a^{\prime}, c^{\prime}$ are new vertices. Thus this family is infinite. It is easy to show that special graphs are 3-connected. It follows from the definition that if $G$ is special and $u v \notin E(G)$ then $G+u v$ is a 3 -connected $M$-circuit. Thus $G+u v$ is globally rigid by Theorems 5.1(a) and 1.4, and $u v$ is critical in $G+u v$. Hence Theorem 7.1 implies that each pair of vertices in a special graph is either globally linked or globally loose:

Theorem 7.2. Let $G$ be special and suppose that $u, v \in V$. Then $\{u, v\}$ is globally loose in $G$ if and only if $u v \notin E$.

Theorem 7.2 implies that Conjecture 5.9 holds for special graphs.

\section{The Number of Equivalent Realizations}

The following folklore result is known to hold in $\mathbb{R}^{d}$. We include a proof for the twodimensional case for the sake of completeness. 
Theorem 8.1. Suppose that $(G, p)$ is a rigid generic framework. Then the number of distinct congruence classes of frameworks which are equivalent to $(G, p)$ is finite.

Proof. Let $D=\sum_{u v \in E}\|p(u)-p(v)\|$ and $B=\left\{x \in \mathbb{R}^{2 n}:\|x\| \leq D\right\}$, where $n=|V|$. We can choose a representative $\left(G, q_{i}\right)$ for each congruence class, such that $\left(G, q_{i}\right)$ is in standard position. Since $G$ is connected, $q_{i} \in B$.

Suppose that there are infinitely many distinct congruence classes of $(G, p)$. Since $B$ is compact, we may choose a sequence of representatives $\left(G, q_{i}\right)$ converging to a limit $(G, q)$. Then $(G, q)$ is equivalent to $(G, p)$ and hence, by Corollary 3.7, $(G, q)$ is quasi-generic. This contradicts the fact that $(G, p)$, and hence $(G, q)$, is rigid since the frameworks $\left(G, q_{i}\right)$ are pairwise non-congruent.

Given a rigid generic framework $(G, p)$, let $h(G, p)$ denote the number of distinct congruence classes of frameworks which are equivalent to $(G, p)$. Given a rigid graph $G$, let $h(G)=\max \{h(G, p)\}$, where the maximum is taken over all generic frameworks $(G, p)$. The graph of Fig. 1 shows that $h(G, p)$ need not be the same for all generic realizations $(G, p)$ of a rigid graph $G$.

Borcea and Streinu [3] investigated the number of realizations of minimally rigid frameworks $(G, p)$ with generic edge lengths. (Note that, by Lemmas 3.4 and 3.5, the edge lengths of $(G, p)$ are generic if and only if there is a generic realization $(G, q)$ with the same edge lengths as $(G, p)$.) They counted the number of realizations up to rigid motions i.e. combinations of translations and rotations of the plane. This number is twice as large as $h(G, p)$ since reflections of the plane are not allowed. Their results imply that $h(G) \leq 4^{n}$ for all rigid graphs $G$. They also construct an infinite family of generic minimally rigid frameworks $(G, p)$ for which $h(G, p)$ has order $12^{n / 3} \sim(2.28)^{n}$.

We shall determine the exact value of $h(G, p)$ for all generic realizations $(G, p)$ of an $M$-connected graph $G=(V, E)$. For $u, v \in V$, let $b(u, v)$ denote the number of components of $G-\{u, v\}$ and put $c(G)=\sum_{u, v \in V}(b(u, v)-1)$.

Theorem 8.2. Let $G$ be an $M$-connected graph. Then $h(G, p)=2^{c(G)}$ for all generic realizations $(G, p)$ of $G$.

Proof. Choose a generic framework $(G, p)$. We use induction on $c(G)$. If $c(G)=0$ then $G$ is 3-connected. It follows from Theorems 5.1(a) and 1.4 that $G$ is globally rigid, and hence $h(G, p)=1=2^{c(G)}$. Hence we may assume that there exists a 2-separation $\left(G_{1}, G_{2}\right)$ in $G$ with $V\left(G_{1}\right) \cap V\left(G_{2}\right)=\{u, v\}$. Let $G_{1}$ and $G_{2}$ denote the cleavage graphs obtained by cleaving $G$ along $\{u, v\}$. Note that $u v \in E\left(G_{i}\right)$ and, by Lemma 5.3(b), $G_{i}$ is $M$-connected, for $1 \leq i \leq 2$. Choosing the 2 -separation so that $G_{1}$ is minimal, we also have that $G_{1}$ is 3-connected (see Lemma 2.8 of [1]) and, by Lemma 3.6 of [13], $c\left(G_{2}\right)=c(G)-1$.

By Theorem 5.7, $\{u, v\}$ is globally linked in $G$. Since $G_{1}$ is globally rigid by Theorem 1.4, each congruence class of $(G, p)$ contains a unique framework $(G, q)$ with $p(x)=$ $q(x)$ for all $x \in V\left(G_{1}\right)$. Letting $p^{\prime}=\left.p\right|_{V\left(G_{2}\right)}$ and $q^{\prime}=\left.q\right|_{V\left(G_{2}\right)}$, we may deduce that the number of distinct congruence classes of $(G, p)$ is equal to the number of distinct 
frameworks $\left(G_{2}, q^{\prime}\right)$ which are equivalent to $\left(G_{2}, p^{\prime}\right)$ and satisfy $q^{\prime}(u)=p^{\prime}(u)$ and $q^{\prime}(v)=p^{\prime}(v)$. The number of such frameworks is $2 h\left(G_{2}, p^{\prime}\right)$, since each congruence class of $\left(G_{2}, p^{\prime}\right)$ contains exactly two such frameworks (which can be obtained from each other by a reflection in the line through $\left.p^{\prime}(u), p^{\prime}(v)\right)$. By induction $h\left(G_{2}, p^{\prime}\right)=2^{c(G)-1}$. Thus $h(G, p)=2^{c(G)}$.

It follows from the proof of the above theorem that if $(G, p)$ is a generic realization of an $M$-connected graph $G$, then we can obtain a representative of each distinct congruence class of frameworks which are equivalent to $(G, p)$ by iteratively applying the following operation to $(G, p)$ : choose a 2-separation $\{u, v\}$ of $G$ and reflect some, but not all, of the components of $G-\{u, v\}$ in the line through the points $p(u), p(v)$.

Theorem 8.2 implies that $h(G, p)$ is the same for all generic realizations of an $M$ connected graph $G$. Note that this statement becomes false if we replace the hypothesis that $G$ is $M$-connected by the weaker hypothesis that $G$ is redundantly rigid. An example is the redundantly rigid graph $G$ obtained from the graph in Fig. 1 by replacing each edge by a copy of $K_{4}$.

Theorem 8.2 also implies that $h(G) \leq 2^{(n-2) / 2-1}$ for all $M$-connected graphs $G$. A family of graphs attaining this bound is a collection of $K_{4}$ 's joined along a common edge.

\section{Acknowledgement}

The first author thanks Steve Donkin for helpful conversations concerning Lemmas 3.1 and 3.2.

\section{References}

1. A.R. Berg and T. Jordán, A proof of Connelly's conjecture on 3-connected circuits of the rigidity matroid, J. Combin. Theory Ser. B. 88 (2003), 77-97.

2. A.R. Berg and T. Jordán, Algorithms for graph rigidity and scene analysis, Proc. 11th Annual European Symposium on Algorithms (ESA) 2003 (G. Di Battista and U. Zwick, eds.), pp. 78-89, Lecture Notes in Computer Science, Vol. 2832, Springer, Berlin, 2003.

3. C. Borcea and I. Streinu, The number of embeddings of minimally rigid graphs, Discrete Comput. Geom. 31 (2004), 287-303.

4. R. Connelly, Rigidity and energy, Invent. Math. 66(1) (1982), 11-33.

5. R. Connelly, On generic global rigidity, in Applied Geometry and Discrete Mathematics, pp. 147-155, DIMACS Series in Discrete Mathematics and Theoretical Computer Science, Vol. 4, American Mathematical Society, Providence, RI, 1991.

6. R. Connelly, Generic global rigidity, Discrete Comput. Geom. 33 (2005), 549-563.

7. T. Eren, D. Goldenberg, W. Whiteley, Y.R. Yang, A.S. Morse, B.D.O. Anderson, and P.N. Belhumeur, Rigidity, computation, and randomization in network localization, Proc. IEEE INFOCOM Conference, Hong-Kong, March 2004, pp. 2673-2684.

8. H. Gluck, Almost all simply connected closed surfaces are rigid, Geometric Topology (Proc. Conf., Park City, Utah, 1974), pp. 225-239, Lecture Notes in Mathematics, Vol. 438, Springer, Berlin, 1975.

9. D.K. Goldenberg, A. Krishnamurthy, W.C. Maness, Y.R. Yang, A. Young, A.S. Morse, A. Savvides, and B.D.O. Anderson, Network localization in partially localizable networks, Proc. IEEE INFOCOM Conference, Miami, March 2005, to appear. 
10. J. Graver, B. Servatius, and H. Servatius, Combinatorial Rigidity, Graduate Studies in Mathematics, Vol. 2, American Mathematical Society, Providence, RI, 1993.

11. B. Hendrickson, Conditions for unique graph realizations, SIAM J. Comput. 21(1) (1992), 65-84.

12. L. Henneberg, Die graphische Statik der starren Systeme, Teubner, Leipzig, 1911; Johnson reprint, London, 1968.

13. B. Jackson and T. Jordán, Connected rigidity matroids and unique realizations of graphs, J. Combin. Theory Ser. B 94 (2005), 1-29.

14. G. Laman, On graphs and rigidity of plane skeletal structures, J. Engrg. Math. 4 (1970), 331-340.

15. L. Lovász and Y. Yemini, On generic rigidity in the plane, SIAM J. Algebraic Discrete Methods 3(1) (1982), 91-98.

16. H. Nagamochi and T. Ibaraki, A linear-time algorithm for finding a sparse $k$-connected spanning subgraph of a $k$-connected graph, Algorithmica 7 (1992), 583-596.

17. J.G. Oxley, Matroid Theory, Oxford University Press, New York, 1992.

18. J.B. Saxe, Embeddability of weighted graphs in $k$-space is strongly NP-hard, Technical Report, Computer Science Department, Carnegie-Mellon University, Pittsburgh, PA, 1979.

19. I. Stewart, Galois Theory, 3rd edn., Chapman and Hall/CRC Press, London/Boca Raton, FL, 2004.

20. W. Whiteley, Some matroids from discrete applied geometry, in Matroid Theory (Seattle, WA, 1995), pp. 171-311, Contemporary Mathematics, Vol. 197, American Mathematical Society, Providence, RI, 1996.

Received March 15, 2005, and in revised form September 9, 2005. Online publication January 20, 2006. 